\title{
ON LIFTING TRANSFORMATION GROUPS
}

\author{
F. RHODES
}

It is known [1] that if $(X, G, \mu)$ is a topological transformation group such that $\pi_{1}(G)=0$, and if $\tilde{X}$ is a covering space of $X$, then there is a unique topological transformation group $(\tilde{X}, G, \tilde{\mu})$ which covers $(X, G, \mu)$. In [2] the fundamental group $\sigma\left(X, x_{0}, G\right)$ of a group of homeomorphisms $G$ of a topological space $X$ is defined, and it is observed in the proof of Theorem 7 of that paper that $\sigma\left(X, x_{0}, G\right)$ acts in a natural way as a group of homeomorphisms of the universal covering space $\tilde{X}$. In this note the relationship between these two results is investigated, and equations defining $\tilde{\mu}$ are found.

The language and notations will be minor modifications of those of [2], together with standard notations for covering spaces. It will be assumed throughout that $X$ is path-connected, locally path-connected, and locally simply connected, that $G$ is a locally pathconnected topological group, and that $(X, G, \mu)$ is a topological transformation group.

First observe that every homeomorphism $g$ of a path-connected space $X$ induces an automorphism of the group $N$ of normal subgroups of $\pi_{1}\left(X, x_{0}\right)$. If $k$ is a path from $g x_{0}$ to $x_{0}$, then the map $[f] \rightarrow[k \rho+g f+k]$ is an automorphism of $\pi_{1}\left(X, x_{0}\right)$. The image of a normal subgroup $\pi_{0}$ is a normal subgroup which is independent of $k$, and which will be denoted by $g_{*} \pi_{0}$. It is easily checked that $g_{*}: N \rightarrow N$ is an automorphism.

Definition 1. A normal subgroup $\pi_{0}$ of $\pi_{1}\left(X, x_{0}\right)$ is said to be invariant under $G$ if for every $g \in G, g_{*} \pi_{0}=\pi_{0}$.

Let $[f ; g]_{\pi_{0}}$ denote the equivalence class of the path $f$ of order $g$ under the equivalence relation

$$
(f ; g) R\left(f^{\prime} ; g^{\prime}\right) \quad \text { iff } g=g^{\prime} \text { and }\left[f+f^{\prime} \rho\right] \in \pi_{0} .
$$

Lemma 1. If $\pi_{0}$ is invariant under $G$, then the set of homotopy classes $[f ; g]_{\pi_{0}}$ with the rule of composition

$$
\left[f_{1} ; g_{1}\right]_{\pi_{0}} *\left[f_{2} ; g_{2}\right]_{\pi_{0}}=\left[f_{1}+g_{1} f_{2} ; g_{1} g_{2}\right]_{\pi_{0}}
$$

forms a group $\sigma_{\pi_{0}}\left(X, x_{0}, G\right)$.

The proof is omitted.

The group $\sigma\left(X, x_{0}, G\right)$ defined in [2] corresponds to the case in which $\pi_{0}$ is the trivial subgroup of $\pi_{1}\left(X, x_{0}\right)$.

Received by the editors April 12, 1967. 
There are two short exact sequences for $\sigma_{\pi_{0}}\left(X, x_{0}, G\right)$, in which the maps correspond to those in the exact sequence for $\sigma\left(X, x_{0}, G\right)$ described in [2].

$$
\begin{aligned}
& 0 \rightarrow \pi_{1}\left(X, x_{0}\right), \pi_{0} \stackrel{i}{\rightarrow} \sigma_{\pi_{0}}\left(X, x_{0}, G\right) \stackrel{j}{\rightarrow} G \rightarrow 0, \\
& 0 \rightarrow \pi_{0} \rightarrow \sigma\left(X, x_{0}, G\right) \rightarrow \sigma_{\pi_{0}}\left(X, x_{0}, G\right) \rightarrow 0 .
\end{aligned}
$$

A basis of open neighbourhoods is defined for $\sigma_{x_{0}}\left(X, x_{0}, G\right)$ as follows. Given $[f ; g]_{\pi_{0}}$ and open neighbourhoods $U\left(g x_{0}\right)$ in $X$ and $V(e)$ in $G$, define $W[f ; g]_{\pi_{0}}$ to be the set of classes $\left[f+f^{\prime} ; g^{\prime}\right]_{\pi_{0}}$ where $g^{\prime} g^{-1} \in V$ and $f^{\prime}$ is a path in $U\left(g x_{0}\right)$ from $g x_{0}$ to $g^{\prime} x_{0}$. Sets of the form of $W$ constitute a basis for a topology on $\sigma_{\pi_{0}}\left(X, x_{0}, G\right)$. Observe that if the isotropy subgroup of $G$ at $x_{0}$ is trivial, then the map $[f ; g]_{\pi_{0}}$ $\rightarrow[f]_{\pi_{0}}$ is an injection of $\sigma_{\pi_{0}}\left(X, x_{0}, G\right)$ into the covering space $\tilde{X}_{\pi_{0}}$.

Proposition 1. With the topology just defined, $\sigma_{\pi_{0}}\left(X, x_{0}, G\right)$ is a topological group.

Proof. A proof of the continuity of the product is sketched. The proof of the continuity of the inverse is similar.

Let elements $\left[f_{1} ; g_{1}\right]_{\pi_{0}}$ and $\left[f_{2} ; g_{2}\right]_{\pi_{0}}$ be given, and let an open neighbourhood $W$ of their product be given in terms of an open neighbourhood $U\left(g_{1} g_{2} x_{0}\right)$ and an open neighbourhood $V(e)$. Then there exist neighbourhoods $U_{i}\left(g_{i} x_{0}\right), V_{i}(e)(i=1,2)$, such that

(i) $V_{1} \times V_{1} \subset V$ and $V_{2} \subset g_{1}^{-1} V_{1} g_{1}$;

(ii) $h \in V_{1}$ and $x^{\prime} \in U_{2}$ imply $h g_{1}\left(x_{0}\right) \in U_{1}, \quad h g_{1} g_{2}\left(x_{0}\right) \in U$ and $h g_{1}\left(x^{\prime}\right) \in U$;

(iii) every loop in $U_{1}$ is nullhomotopic in $X$;

(iv) every pair of elements in $V_{2} g_{1}$ can be joined by a path in $V_{1} g_{1}$. Now let $g_{i}^{\prime} \in V_{2} g_{i}$ and let $f_{i}^{\prime}$ be a path in $U_{i}$ from $g_{i} x_{0}$ to $g_{i}^{\prime}\left(x_{0}\right)$ $(i=1,2)$. Let $\phi$ be a path in $V_{1} g_{1}$ from $g_{1}$ to $g_{1}^{\prime}$. Define a map $\Phi: I \times I$ $\rightarrow X$ by $\Phi(s, t)=\phi(s) f_{2}(t)$, and set $\Phi(s, 0)=\phi_{0}(s), \Phi(s, 1)=\phi_{1}(s)$. Then $f_{1}^{\prime}+\phi_{0} \rho$ is nullhomotopic and $\phi_{0}+g_{1}^{\prime} f_{2} \sim g_{1} f_{2}+\phi_{1}$. Hence $\left[f_{1}+f_{1}^{\prime}+g_{1}^{\prime}\left(f_{2}+f_{2}^{\prime}\right) ; g_{1}^{\prime} g_{2}^{\prime}\right]_{\pi_{0}}=\left[f_{1}+g_{1} f_{2}+\phi_{1}+g_{1}^{\prime} f_{2}^{\prime} ; g_{1}^{\prime} g_{2}^{\prime}\right]_{\pi_{0}} \in W$.

Relative to this topology, the homomorphism

$$
\lambda_{*}: \sigma_{\pi_{0}}\left(X, x_{0}, G\right) \rightarrow \sigma_{\pi_{0}}\left(X, x_{1}, G\right)
$$

induced by a path $\lambda$ from $x_{0}$ to $x_{1}$ is continuous. Moreover, if $(\phi, \psi)$ : $(X, G, \mu) \rightarrow\left(X^{\prime}, G^{\prime}, \mu^{\prime}\right)$ is a homomorphism of the topological thtransformation groups, then the induced homomorphism

$$
(\phi, \psi)_{*}: \sigma\left(X, x_{0}, G\right) \rightarrow \sigma\left(X^{\prime}, x_{0}^{\prime}, G^{\prime}\right)
$$

is continuous. 
If $\pi^{1}$ and $\pi^{2}$ are normal subgroups of $\pi_{1}\left(X, x_{0}\right)$ which are invariant under $G$, and if $\pi^{1} \subset \pi^{2}$, then the map

$$
\tilde{\mu}: \sigma_{\pi^{1}}\left(X, x_{0}, G\right) \times \tilde{X}_{\pi^{2}} \rightarrow \tilde{X}_{\pi^{2}}, \quad \tilde{\mu}:\left([f ; g]_{\pi^{1}},\left[f^{\prime}\right]_{\pi^{2}}\right) \rightarrow\left[f+g f^{\prime}\right]_{\pi^{2}},
$$

is,well defined.

Proposition 2. With the action $\tilde{\mu}$ just defined, $\left(\tilde{X}_{\pi^{2}}, \sigma_{\pi^{1}}\left(X, x_{0}, G\right), \tilde{\mu}\right)$ is a topological transformation group.

Proof. The proof of the continuity of $\tilde{\mu}$ is similar to the proof of Proposition 1.

All the natural diagrams commute. In particular, $\tilde{\mu}$ covers $\mu$, and the action of $\tilde{\mu}_{\pi_{0}}\left(X, x_{0}, G\right)$ on any covering space derives from its action on $\tilde{X}_{\pi_{0}}$.

It is interesting to note that $\sigma\left(\tilde{X}_{\tau_{0}}, \sigma_{\tau_{0}}\left(X, x_{0}, G\right)\right) \cong \sigma\left(X, x_{0}, G\right)$ the map $\left[F ;[f ; g]_{\pi_{0}}\right] \rightarrow[p F ; g]$ is a homomorphism, and that it is an isomorphism follows from the application of the 5-lemma to the sequences

$$
\begin{aligned}
& 0 \rightarrow \pi_{1}\left(\tilde{X}_{\pi_{0}}, \tilde{x}_{0}\right) \rightarrow \sigma\left(\tilde{X}_{\pi_{0}}, \sigma_{\pi_{0}}\left(X, x_{0}, G\right)\right) \rightarrow \sigma_{\pi_{0}}\left(X, x_{0}, G\right) \rightarrow 0, \\
& 0 \rightarrow \pi_{0} \rightarrow \sigma\left(X, x_{0}, G\right) \rightarrow \sigma_{\pi_{0}}\left(X, x_{0}, G\right) \rightarrow 0 .
\end{aligned}
$$

In $\S 9$ of [2] the concept of a family of preferred paths is defined. One can confirm that $X$ admits a family of preferred paths at $x_{0}$ if and only if the following sequence splits.

$$
0 \rightarrow \pi_{1}\left(X, x_{0}\right) \stackrel{i}{\rightarrow} \sigma\left(X, x_{0}, G\right) \stackrel{j}{\rightarrow} G \rightarrow 0 .
$$

In this case, if $\boldsymbol{k}: G \rightarrow \sigma\left(X, x_{0}, G\right)$ is a splitting homomorphism and $\tilde{\mu}: \sigma\left(X, x_{0}, G\right) \times \tilde{X}_{\pi_{0}} \rightarrow \tilde{X}_{x_{0}}$, then $\tilde{\mu}(\mathrm{id}, \boldsymbol{k})$ defines an action of $G$ as a group of homeomorphisms of $\tilde{X}_{\pi_{0}}$. However, since $k$ need not be continuous, this is no guarantee that there is a topological transformation group $\left(\tilde{X}_{\pi_{0}}, G, \mu_{1}\right)$ which covers $(X, G, \mu)$.

Definition 2. The group $\sigma\left(X, x_{0}, G\right)$ will be said to admit a continuous split extension if there is a continuous homomorphism $k: G \rightarrow \sigma\left(X, x_{0}, G\right)$ such that $j k=$ id.

Lemma 2. If $\sigma\left(X, x_{0}, G\right)$ admits a continuous split extension then it is topologically the product of $G$ and the discrete space $\pi_{1}(X)$.

Proof. Since $X$ is locally simply connected, the topology for $\pi_{1}\left(X, x_{0}\right)$ induced by its inclusion in $\tilde{X}$ is discrete. The proof now follows from a study of the map $([f], g) \rightarrow[f+k g \rho ; g]$.

The group $G$ acts on itself by left translation, and if it is a pathconnected group we can ask whether $\sigma(G, e, G)$ admits a continuous split extension. 
Proposition 3. The group $\sigma(G, e, G)$ admits a continuous split extension if and only if $\pi_{\mathbf{1}}(G, e)=0$.

Proof. The sufficiency of the condition is obvious. Since the isotropy subgroup of $G$ at $e$ is trivial, $\sigma(G, e, G)$ can be regarded as a subspace of the universal covering group $\widetilde{G}$. Hence a map $k: G$ $\rightarrow \sigma(G, e, G)$ can be thought of as a covering map of the identity map of $G$; whence $\pi_{1}(G)=p_{*} \pi_{1}(\tilde{G}, \tilde{e})=0$.

Now we can prove the proposition from which the Lima result will follow. Define $\mu_{x_{0}}: G \rightarrow x, \mu_{x_{0}}(g)=\mu\left(g, x_{0}\right)$.

Proposition 4. If $\sigma\left(X, x_{0}, G\right)$ admits a continuous split extension, and $\pi_{0}$ is invariant under $G$, then there exists a topological transformation group $\left(\tilde{X}_{\pi_{0}}, G, \mu_{1}\right)$ which covers $(X, G, \mu)$. If $\mu_{x_{0 *}} \pi_{1}(G, e) \subset \pi_{0}$, then the covering map $\mu_{1}$ is unique.

Proof. The existence of the continuous map $\mu_{1}$ follows from the discussion above. Its uniqueness follows from standard covering theorems.

Corollary. If $\pi_{1}(G, e)=0$ and $\pi_{0}$ is invariant under $G$, then there exists a unique topological transformation group $\left(\tilde{X}_{\pi_{0}}, G, \mu_{1}\right)$ which covers $(X, G, \mu)$.

Proof. Given $g$, let $l_{g}$ be a path in $G$ from $g$ to $e$, and let $\mu_{x_{0}} l_{g}=k_{g}$. Then $g \rightarrow\left[k_{\theta} \rho ; g\right]$ is a continuous splitting map.

The equation defining $\mu_{1}$ is

$$
\mu_{1}:\left(g,[f]_{\pi_{0}}\right) \rightarrow\left[k_{g} \rho+g f\right]_{\pi_{0}} .
$$

That this map is well defined even if $\pi_{0}$ is not invariant under $G$ follows from an argument similar to that used to prove Theorem 7 of [2]. It is continuous, the proof being similar to that of Proposition 1. Thus, subject to the standing conditions on $X$ and $G$, we have the following result.

Proposition 5. If $\pi_{1}(G, e)=0$ and $\tilde{X}$ is any covering space of $X$, then there is a unique topological transformation group $\left(\tilde{X}, G, \mu_{1}\right)$ which covers $(X, G, \mu)$, and the action of $\mu_{1}$ is defined by the equation above.

\section{REFERENCES}

1. E. L. Lima, Common singularities of commuting vector fields on 2-manifolds, Comment. Math. Helv. 39 (1964), 97-110.

2. F. Rhodes, On the fundamental group of a transformation group, Proc. London Math. Soc. (3) 16 (1966), 635-650.

WESLEYAN UNIVERSITY AND

University of Southampton, England 